\title{
Transporte intra-hospitalar de pacientes internados em UTI Neonatal: fatores de risco para intercorrências
}

\author{
Intra-hospital transport of neonatal intensive care patients: risk factors for complications
}

Anna Luiza P. Vieira ${ }^{1}$, Ruth Guinsburg², Amélia Miyashiro N. Santos ${ }^{3}$, Clóvis de Araújo Peres ${ }^{4}$, Mayra Ivanoff Lora ${ }^{5}$, Milton Harumi Miyoshi $^{6}$

\section{RESUMO}

Objetivo: Estudar os fatores associados à hipotermia e ao aumento da necessidade de oxigênio e/ou suporte ventilatório durante o transporte intra-hospitalar de pacientes internados em Unidade de Terapia Intensiva neonatal.

Métodos: Estudo prospectivo de todos os pacientes internados na unidade neonatal que necessitaram de transporte intra-hospitalar de janeiro de 1997 a dezembro de 2000, entre segundas-feiras e sextas-feiras, das $8 \mathrm{~h}$ às $17 \mathrm{~h}$. Fatores associados à hipotermia e ao aumento da necessidade de oxigênio e/ou de suporte ventilatório durante e até duas horas após o transporte foram estudados por meio de regressão logística.

Resultados: Foram realizados 502 transportes no período. Os pacientes tinham em média $2.000 \mathrm{~g}, 35$ semanas de idade gestacional ao nascer e 22 dias de vida. As principais indicações do transporte foram: cirurgia e realização de exames de imagem. A hipotermia ocorreu em $17 \%$ dos transportes e o aumento da necessidade de oxigênio e/ou de suporte ventilatório em $7 \%$. Fatores associados à hipotermia foram: duração do transporte $>3 \mathrm{~h}(\mathrm{OR}=2,1$; IC95\%=1,2-3,6), presença de malformações neurológicas $(\mathrm{OR}=1,7 ; \mathrm{IC} 95 \%=1,1-2,5)$, transporte realizado em $1997(\mathrm{OR}=1,7 ; \mathrm{IC} 95 \%=1,1-2,6)$ e peso no transporte $>3.500 \mathrm{~g}(\mathrm{OR}=0,3$; IC95\%=0,16-0,68). Fatores de risco para o aumento da necessidade de oxigênio e/ou de suporte ventilatório foram: idade gestacional ao nascimento em semanas $(\mathrm{OR}=0,9 ; \mathrm{IC} 95 \%=0,8-0,9)$, idade em dias no transporte $(\mathrm{OR}=1,0$; IC95\% = 1,0-1,1) e presença de malformações gastrintestinais e geniturinárias $(\mathrm{OR}=3,1$; IC95\%=1,6-6,2).

Conclusões: As intercorrências relativas ao transporte intra-hospitalar são freqüentes nos neonatos em UTI e estão associadas às condições dos pacientes e dos transportes.

Palavras-chave: transporte de pacientes; recém-nascido; unidades de terapia intensiva.

\footnotetext{
${ }^{1}$ Neonatologista assistente e mestre em Pediatria pela Disciplina de Pediatria Neonatal do Departamento de Pediatria da Escola Paulista de Medicina da Universidade Federal de São Paulo (Unifesp-EPM)

2Professora titular da Disciplina de Pediatria Neonatal do Departamento de Pediatria da Unifesp-EPM

${ }^{3}$ Professora associada da Disciplina de Pediatria Neonatal do Departamento de Pediatria da Unifesp-EPM

${ }^{4}$ Professor titular do Departamento de Medicina Preventiva da UnifespEPM

${ }^{5}$ Estagiária do Departamento de Medicina Preventiva da Unifesp-EPM
}

\section{ABSTRACT}

Objective: Evaluate factors associated with hypothermia and increased need of oxygen and/or ventilatory support during intra-hospital transport of neonatal intensive care patients.

Methods: Prospective study of infants admitted to a single neonatal unit in need of at least one intra-hospital transport during weekdays, from 8:00AM to 05:00PM, from January 1997 to December 2000. Factors associated with hypothermia and increased need of oxygen and/or need of ventilatory support during and up to two hours after transport were studied by regression analyses.

Results: During the study period, 502 transports were analyzed. At the time of transport, the neonates had a mean weight and gestational age of, respectively, 2,000g and 35 weeks, and they were 22 days old. The main reasons for transport were: surgery and image exams. Hypothermia occurred in $17 \%$ of the transported infants and increased need of oxygen and/or ventilatory support in $7 \%$. Factors associated with hypothermia were: duration of transportation $>3 \mathrm{~h}(\mathrm{OR}=2.1 ; 95 \% \mathrm{CI}=1.2$ 3.6), neurologic malformation ( $\mathrm{OR}=1.7 ; 95 \% \mathrm{CI}=1.1-2.5)$, transport performed in $1997(\mathrm{OR}=1.7 ; 95 \% \mathrm{CI}=1.1-2.6)$ and weight at time of transport $>3,500 \mathrm{~g}(\mathrm{OR}=0.3 ; 95 \% \mathrm{CI}=0.16$ 0.68). Factors associated with increased need of oxygen and/or ventilation support were: gestational age at birth in weeks $(\mathrm{OR}=0.9 ; 95 \% \mathrm{CI}=0.8-0.9)$, age in days at transportation $(\mathrm{OR}=1.0 ; 95 \% \mathrm{CI}=1.0-1.1)$ and presence of gastrointestinal and genitourinary malformation $(\mathrm{OR}=3.1 ; 95 \% \mathrm{CI}=1.6-6.2)$.

Conclusions: Complications related to neonatal intrahospital transports are frequent and associated with the patients' characteristics and transport conditions.

Key-words: transportation of patients; infant, newborn; intensive care units.

${ }^{6}$ Professor assistente da Disciplina de Pediatria Neonatal do Departamento de Pediatria da Unifesp-EPM

Endereço para correspondência:

Ruth Guinsburg

Rua Dr. Diogo de Faria, 764

CEP 04037-002 - São Paulo/SP

E-mail: ruthgbr@netpoint.com.br

Recebido em: 17/5/2007

Aprovado em: 10/7/2007 


\section{Introdução}

O transporte intra-hospitalar neonatal ocorre quando os pacientes internados em unidade neonatal são transportados para realizar alguma intervenção cirúrgica ou procedimento diagnóstico ${ }^{(1-5)}$. Estes transportes podem se constituir em risco adicional para o neonato criticamente doente e, por isso, devem ser considerados como uma extensão dos cuidados realizados na Unidade de Terapia Intensiva (UTI). Vale lembrar que o transporte intra-hospitalar ocorre com grande freqüência e, para a sua realização, são necessários equipamentos e pessoal habilitado, de forma similar à necessária para o transporte entre hospitais ${ }^{(1)}$.

As intercorrências passíveis de ocorrer durante o transporte intra-hospitalar podem se associar às alterações fisiológicas e/ou clínicas do próprio paciente e a problemas ligados aos equipamentos e/ou à equipe de transporte. As intercorrências clínicas neste tipo de transporte são bastante freqüentes em pacientes adultos, descrevendo-se a presença de alterações hemodinâmicas ${ }^{(6-7)}$, bradicardia e hipoxemia, problemas com a pressão arterial e a freqüência respiratória ${ }^{(8)}$, além de alterações dos gases sangüíneos em adultos intubados ${ }^{(9)}$.

Os estudos dos problemas relativos ao transporte intra-hospitalar de neonatos são escassos ${ }^{(6)}$, mas pode haver alterações significativas dos sinais vitais como temperatura corporal, freqüência cardíaca e respiratória, pressão arterial, saturação de oxigênio, pressão parcial de oxigênio e do gás carbônico. Ressalta-se que, mesmo com o adequado preparo do recémnascido, as condições inerentes ao transporte, tais como barulho excessivo, vibrações e alterações de temperatura comprometem a estabilidade clínica do paciente ${ }^{(10)}$. Neste contexto, o objetivo do presente estudo foi verificar os fatores associados à hipotermia e ao aumento da necessidade de oxigênio e/ou suporte ventilatório durante e até duas horas após o transporte intra-hospitalar de pacientes internados em UTI neonatal.

\section{Métodos}

Estudo prospectivo foi realizado na unidade neonatal do Hospital São Paulo da Universidade Federal de São Paulo (HSP/Unifesp). O levantamento de dados foi iniciado após a aprovação do projeto pelo Comitê de Ética em Pesquisa do HSP/Unifesp, garantindo-se a confidencialidade das informações obtidas.

Foram incluídos no estudo todos os transportes intra-hospitalares de pacientes internados na UTI Neonatal do HSP/ Unifesp, no período de janeiro de 1997 a dezembro de 2000, efetuados pela equipe de transporte da unidade. Essa equipe era composta por um pediatra no segundo ano de especialização em neonatologia, treinado em reanimação neonatal e capaz de realizar procedimentos de suporte avançado de vida, e por uma enfermeira ou auxiliar de enfermagem da própria unidade, com conhecimento da prática de terapia intensiva neonatal. Tal equipe estava disponível de segunda-feira a sexta-feira, das $8 \mathrm{~h}$ às $17 \mathrm{~h} \mathrm{e}$, portanto, foram incluídos apenas os transportes realizados nesse período, durante os quatros anos avaliados. $\mathrm{O}$ mesmo paciente pode ter sido analisado diversas vezes no estudo, desde que ele fosse transportado em ocasiões diferentes durante a internação hospitalar. Durante este período, os pacientes foram sempre transportados em incubadora de transporte de dupla parede, com um oxímetro de pulso e uma bomba de infusão perfusora alimentados por bateria acoplados à incubadora. A ventilação era feita de forma manual, com balão auto-inflável com capacidade de fornecer oxigênio a 100\%, 40\% ou ar ambiente. Os equipamentos básicos disponíveis para o transporte neonatal se mantiveram constantes durante o período de estudo. Nenhum transporte executado sob as condições acima relatadas foi excluído da pesquisa.

A coleta de dados foi feita de forma prospectiva por meio de fichas preenchidas ao final de cada transporte pelo neonatologista responsável pelo transporte. Coletaram-se dados dos pacientes (idade gestacional ao nascer, dias de vida no transporte, peso ao nascer e no transporte, gênero e doença clínica pré-existente) e dos transportes (ano de sua realização, indicações e duração em minutos a partir da saída da criança da unidade neonatal até seu retorno à unidade). Os sinais vitais foram obtidos ao início do transporte, durante e ao retorno na unidade. A temperatura era mensurada por termômetro digital em região axilar a cada dez minutos durante o transporte, sendo considerada hipotermia a temperatura inferior a $36^{\circ} \mathrm{C}$ e hipertermia quando superior a $37,5^{\circ} \mathrm{C}^{(11)}$. A saturação de oxigênio se baseou nos registros do oxímetro de pulso durante todo o transporte. Finalmente, analisou-se a indicação de oxigenoterapia e/ou de ventilação mecânica em pacientes que previamente não faziam uso dessas intervenções ou a necessidade de aumentar a concentração de oxigênio e/ou dos parâmetros de suporte ventilatório nos pacientes que faziam uso prévio destas medidas de suporte. Definiu-se como intercorrência relacionada ao transporte a presença de hipotermia e/ou necessidade de aumento do suporte respiratório manifestas desde o início do transporte até duas horas após o retorno do paciente à UTI neonatal.

$\mathrm{Na}$ análise dos resultados, as variáveis numéricas foram descritas em média e desvio padrão ou mediana e variação. As variáveis categóricas foram relatadas em frequiência dos eventos estudados. Para avaliar os fatores associados à presença 
de hipotermia e à necessidade de aumentar a concentração de oxigênio e/ou os parâmetros do suporte ventilatório, foi feita a análise de regressão logística. Como variáveis independentes, escolheram-se aquelas ligadas às características dos pacientes transportados e do próprio transporte $\operatorname{com} p<0,20$ à análise univariada. A modelagem foi feita com a retirada uma a uma das variáveis não significantes, até obter um modelo final $\operatorname{com} p<0,05$ para a razão de verossimilhança. Os dados foram analisados por meio do SPSS 11.0.

\section{Resultados}

Foram analisados 502 transportes, sendo 129 em 1997, 132 em 1998, 131 em 1999 e 110 em 2000. Vários pacientes foram submetidos a mais de um transporte, dependendo da doença de base e da indicação do transporte (Tabela 1). Como os pacientes transportados mais de uma vez foram submetidos a esse procedimento por razões distintas, em diferentes períodos de sua permanência intra-hospitalar, os dados estão referidos por transporte realizado e não por paciente.

As características demográficas dos pacientes submetidos ao transporte intra-hospitalar estão descritas na Tabela 2. O peso ao nascer de toda a população transportada variou de 600 a $5.200 \mathrm{~g}$. A idade gestacional em semanas variou entre 24 semanas e seis dias a 40 semanas e um dia, sendo, em média, próxima a 35 semanas em todos os anos. Em relação à idade na época do transporte, esta variou de um dia até depois do primeiro ano de vida, sendo que o paciente de maior idade foi transportado em 2000, com 495 dias de vida e 8.000g.

As doenças relacionadas à indicação do transporte intrahospitalar foram divididas em sete grupos: respiratórias, cardiovasculares, infecciosas, doenças gastrintestinais, malformações geniturinárias, alterações neurológicas e síndromes genéticas ou cromossômicas. Como demonstrado na Tabela 3, as doenças neurológicas constituíram a principal indicação de transporte intra-hospitalar em todos os anos do estudo.

De maneira paralela às entidades clínicas associadas à indicação de transporte, os seguintes procedimentos, em todo o período de estudo, necessitaram de transportes (Tabela 4): exames de diagnóstico por imagem em $322(64 \%)$ transportes, procedimentos cirúrgicos em 120 (24\%) e eletroencefalograma em 42 (8\%).

Em relação à duração do transporte, observou-se que, em média, este foi superior a uma hora em todos os anos de estudo, variando entre 20 minutos e cinco horas. Em

Tabela 1 - Número (porcentagem) de pacientes submetidos a um, dois ou mais transportes intra-hospitalares nos anos de 1997 a 2000

\begin{tabular}{lcccr}
\hline & $\mathbf{1 9 9 7}$ & $\mathbf{1 9 9 8}$ & $\mathbf{1 9 9 9}$ & $\mathbf{2 0 0 0}$ \\
& $\mathbf{n = 8 0}$ & $\mathbf{n = 8 1}$ & $\mathbf{n = 7 1}$ & \multicolumn{1}{c}{$\mathbf{n = 5 9}$} \\
\hline Um transporte & $52(65 \%)$ & $52(64 \%)$ & $32(45 \%)$ & $33(56 \%)$ \\
Dois transportes & $14(17 \%)$ & $18(22 \%)$ & $27(38 \%)$ & $9(15 \%)$ \\
Três ou mais transportes & $14(17 \%)$ & $11(14 \%)$ & $12(17 \%)$ & $17(29 \%)$ \\
\hline
\end{tabular}

Tabela 2 - Características demográficas dos pacientes submetidos ao transporte intra-hospitalar entre 1997 e 2000

\begin{tabular}{|c|c|c|c|c|c|}
\hline & $\begin{array}{c}1997 \\
n=129\end{array}$ & $\begin{array}{c}1998 \\
n=132\end{array}$ & $\begin{array}{c}1999 \\
n=131\end{array}$ & $\begin{array}{c}2000 \\
n=110\end{array}$ & $\begin{array}{c}\text { Total } \\
\mathrm{n}=502\end{array}$ \\
\hline \multicolumn{6}{|l|}{ PN (g) } \\
\hline Mediana & 2.640 & 2.300 & 1.710 & 1.925 & 2.155 \\
\hline $\begin{array}{c}\text { Min/máx } \\
\text { Id Gest (sem) }\end{array}$ & $650 / 4.950$ & $600 / 4.330$ & $670 / 3.920$ & $785 / 5.200$ & $600 / 5.200$ \\
\hline Mediana & 36,7 & 34,9 & 35,0 & 34,1 & 35,4 \\
\hline Min/máx & $24,6 / 39,3$ & $24,9 / 40,1$ & $25,4 / 40,0$ & $25,7 / 39,3$ & $24,6 / 40,1$ \\
\hline \multicolumn{6}{|l|}{ Sexo feminino } \\
\hline n (\%) & $63(48,8)$ & $84(63,6)$ & $81(61,8)$ & $68(61,8)$ & $296(58,9)$ \\
\hline \multicolumn{6}{|c|}{ DV Transporte } \\
\hline Mediana & 13 & 23 & 21 & 30 & 22 \\
\hline Min/máx & $1 / 163$ & $1 / 221$ & $1 / 270$ & $1 / 495$ & $1 / 495$ \\
\hline \multicolumn{6}{|c|}{ Peso transp $(\mathrm{g})$} \\
\hline Mediana & 2.640 & 2.545 & 2.280 & 2.820 & 2.560 \\
\hline Min/máx & $811 / 4.995$ & $820 / 6.530$ & $770 / 5.940$ & $836 / 8.000$ & $770 / 8.000$ \\
\hline
\end{tabular}

PN - peso ao nascer; Id Gest - idade gestacional ao nascer; DV - dias de vida; P transp - peso no dia do transporte 
1997, a duração média do transporte foi $109 \pm 53$ minutos;

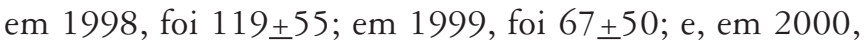
$89 \pm 53$ minutos.

A freqüência de hipotermia durante e até duas horas após o transporte foi elevada, ocorrendo em 87 (17\%) dos 502 trans- portes estudados, decrescendo no decorrer dos anos de estudo (1997: 25\%, 1998: 17\%, 1999: 17\% e 2000: 9\% dos transportes). Já a presença de hipertermia foi excepcional em todos os anos analisados, aparecendo em quatro $(0,8 \%)$ pacientes transportados.

Tabela 3 - Principais entidades mórbidas relacionadas à indicação do transporte intra-hospitalar nos pacientes transportados entre 1997 e 2000, expressas em número de recém-nascidos e porcentagem

\begin{tabular}{|c|c|c|c|c|c|}
\hline & $\begin{array}{c}1997 \\
n=129\end{array}$ & $\begin{array}{c}1998 \\
n=132\end{array}$ & $\begin{array}{c}1999 \\
n=131\end{array}$ & $\begin{array}{c}2000 \\
n=110\end{array}$ & $\begin{array}{c}\text { Total } \\
n=502\end{array}$ \\
\hline Respiratórias & $17(13,1)$ & $13(9,8)$ & $29(22,1)$ & $25(22,7)$ & $84(16,7)$ \\
\hline SDR & $6(4,6)$ & - & $4(3,0)$ & $3(2,7)$ & $13(2,6)$ \\
\hline Apnéia & $8(6,2)$ & $6(4,5)$ & $9(6,9)$ & $8(7,3)$ & $31(6,2)$ \\
\hline SAM & $2(1,5)$ & $1(0,8)$ & - & - & $3(0,6)$ \\
\hline DBP & $1(0,8)$ & $6(4,5)$ & $16(12,2)$ & $14(12,7)$ & $37(7,3)$ \\
\hline Cardiovasculares & $7(3,9)$ & $11(8,3)$ & $13(9,9)$ & $18(16,3)$ & $49(9,8)$ \\
\hline Malformações & $5(3,9)$ & $6(4,5)$ & $6(4,6)$ & $6(5,4)$ & $23(4,6)$ \\
\hline PCA & $2(1,5)$ & $5 \quad(3,8)$ & $7(5,3)$ & $12(10,9)$ & $26(5,2)$ \\
\hline Infecções & $15(11,6)$ & $2(1,6)$ & $13(9,9)$ & $1(0,9)$ & $31(6,2)$ \\
\hline Sepse & $5(3,9)$ & $1(0,8)$ & $6(4,6)$ & - & $12(2,4)$ \\
\hline Meningite & $6(4,6)$ & $1(0,8)$ & $4(3,0)$ & $1(0,9)$ & $12(2,4)$ \\
\hline Infecção congênita & $4(3,1)$ & - & $3(2,3)$ & - & $7(1,4)$ \\
\hline Gastrintestinais & $11(8,5)$ & $25(18,9)$ & $5(3,8)$ & $8(7,2)$ & $49(9,8)$ \\
\hline ECN & $6(4,6)$ & $4 \quad(3,0)$ & $1(0,8)$ & $3(2,7)$ & $14(2,8)$ \\
\hline Malformações & $5(3,9)$ & $21(15,9)$ & $4(3,0)$ & $5(4,5)$ & $35(7,0)$ \\
\hline \multicolumn{6}{|l|}{ Genitourinárias } \\
\hline Malformações & - & $13(9,8)$ & $7(5,8)$ & $6(5,4)$ & $26(5,2)$ \\
\hline Neurológicas & $68(52,7)$ & $63(47,7)$ & $55(41,9)$ & $34(30,9)$ & $220(43,8)$ \\
\hline Asfixia & $10(7,7)$ & $2(1,5)$ & $3(2,3)$ & $3(2,7)$ & $18(3,6)$ \\
\hline Convulsão & $4(3,1)$ & $3(2,3)$ & $2(1,5)$ & $7(6,4)$ & $16(3,2)$ \\
\hline HPIV & $2(1,6)$ & $15(11,3)$ & $26(19,8)$ & $5(4,5)$ & $48(9,6)$ \\
\hline Malformações & $52(40,3)$ & $43(32,6)$ & $24(18,3)$ & $19(17,3)$ & $138(27,4)$ \\
\hline Síndromes genéticas & $1(0,8)$ & $2(1,5)$ & $8(6,1)$ & $7(6,4)$ & $18(3,6)$ \\
\hline Outras & $10(8,4)$ & $3(2,3)$ & $1(0,8)$ & $11(10,0)$ & $25(5,0)$ \\
\hline
\end{tabular}

SDR - síndrome do desconforto respiratório; SAM - síndrome de aspiração de mecônio; DBP - displasia broncopulmonar; PCA - persistência do canal arterial; ECN - enterocolite necrosante; HPIV - hemorragia peri-intraventricular

Tabela 4 - Procedimento que indicou o transporte intra-hospitalar dos pacientes nos anos de 1997 a 2000, expresso em número de transportes (porcentagem)

\begin{tabular}{|c|c|c|c|c|c|}
\hline & $\begin{array}{c}1997 \\
n=129\end{array}$ & $\begin{array}{c}1998 \\
n=132\end{array}$ & $\begin{array}{c}1999 \\
n=131\end{array}$ & $\begin{array}{c}2000 \\
n=110\end{array}$ & $\begin{array}{c}\text { Total } \\
n=502\end{array}$ \\
\hline Cirurgia & $44(34 \%)$ & $35(27 \%)$ & $16(12 \%)$ & $25(23 \%)$ & $120(24 \%)$ \\
\hline Ultra-som & $10(8 \%)$ & $8(6 \%)$ & $54(41 \%)$ & $6(6 \%)$ & $78(16 \%)$ \\
\hline Tomografia & $27(21 \%)$ & $25(19 \%)$ & $26(20 \%)$ & $14(13 \%)$ & $92(18 \%)$ \\
\hline Ressonância & 7 (5\%) & $4(3 \%)$ & - & $3(3 \%)$ & $14(3 \%)$ \\
\hline Radioisótopo & - & $6(5 \%)$ & $4(3 \%)$ & $1(1 \%)$ & $11(2 \%)$ \\
\hline Ecocardiograma & $5(4 \%)$ & $8(6 \%)$ & $8(6 \%)$ & $15(14 \%)$ & $36(7 \%)$ \\
\hline Radiologia & $19(15 \%)$ & $24(6 \%)$ & $18(14 \%)$ & $30(27 \%)$ & $91(18 \%)$ \\
\hline EEG & $12(9 \%)$ & $21(16 \%)$ & $1(1 \%)$ & $8(7 \%)$ & $42(8 \%)$ \\
\hline Outras & $5(4 \%)$ & $1(1 \%)$ & $4(3 \%)$ & $8(7 \%)$ & $18(4 \%)$ \\
\hline
\end{tabular}

EEG - eletroencefalograma 
Tabela 5 - Fatores associados à presença de hipotermia durante e até duas horas após transporte intra-hospitalar

\begin{tabular}{lcc}
\hline & OR & IC95\% \\
\hline Duração >180 minutos & 2,10 & $1,21-3,63$ \\
Doenças neurológicas ou genéticas & 1,68 & $1,11-2,54$ \\
Transporte no ano 1997 & 1,68 & $1,07-2,60$ \\
Peso no transporte $>3.500 \mathrm{~g}$ & 0,34 & $0,16-0,68$ \\
1.500g $<$ PT <1.999g & 0,52 & $0,27-0,98$ \\
Transporte no ano 2000 & 0,53 & $0,29-0,97$ \\
\hline
\end{tabular}

PT - peso no transporte

Quanto ao suporte respiratório, observou-se que os pacientes iniciaram e terminaram o transporte com mediana de fração inspirada de oxigênio de 0,21 . Houve necessidade de iniciar a oferta de oxigênio inalatório durante o transporte em 15 (3\%) dos 502 transportes realizados. Da mesma forma, houve indicação de iniciar a ventilação mecânica em 38 (8\%) dos 502 transportes realizados. Desses 38, 30 (79\%) haviam sido transportados para o centro cirúrgico e os pacientes foram aí intubados para a anestesia e execução de procedimentos variados. $\mathrm{O}$ aumento da necessidade de oxigênio e/ou de suporte ventilatório para os pacientes já intubados durante e até duas horas após o transporte foi notado em 33 casos, permanecendo a incidência de tal intercorrência entre 5 e $8 \%$ dos transportes realizados, nos diversos anos do estudo.

O modelo final de regressão logística para explicar a ocorrência de hipotermia durante e até duas horas após o transporte intra-hospitalar na população estudada mostrou associação com as seguintes variáveis: peso ao transporte, entidade clínica de base, duração do transporte e ano de realização do transporte (Tabela 5). De maneira geral, pacientes com maior peso ao nascer e transportados no ano de 2000 apresentaram menor risco de hipotermia relacionada ao transporte, enquanto aqueles com doenças neurológicas ou síndromes genéticas, cujo transporte foi realizado no ano inicial do estudo e durou mais que três horas, apresentaram maior chance de hipotermia.

Com relação aos fatores de risco e proteção para o aumento de necessidade de oxigênio e/ou suporte ventilatório, observou-se a associação com entidade clínica de base, idade gestacional e idade ao transporte, (Tabela 6). O risco de aumento de necessidade de oxigênio e/ou suporte ventilatório foi mais elevado para neonatos de menor idade gestacional, maior idade pós-natal e portadores de alterações gastrintestinais e geniturinárias.

\section{Discussão}

Por que realizar um trabalho sobre o transporte intra-hospitalar, já que se trata de uma prática usual, do dia-a-dia de
Tabela 6 - Fatores associados ao aumento da necessidade de oxigênio e/ou do suporte ventilatório durante e até duas horas após transporte intra-hospitalar

\begin{tabular}{lcc}
\hline & OR & IC95\% \\
\hline $\begin{array}{l}\text { Presença de alterações } \\
\text { GI e GU }\end{array}$ & 3,15 & $1,61-6,17$ \\
$\begin{array}{l}\text { Idade ao transporte } \\
\text { em dias }\end{array}$ & 1,01 & $1,00-1,02$ \\
$\begin{array}{l}\text { Idade gestacional } \\
\text { em semanas }\end{array}$ & 0,88 & $0,82-0,96$ \\
\hline
\end{tabular}

$\mathrm{GI}$ - gastrintestinal; GU - geniturinário

grande parte dos serviços neonatais? Várias respostas podem ser dadas: primeiro, esses recém-nascidos podem permanecer "em transporte" por períodos relativamente longos, necessitando dos mesmos cuidados recebidos na unidade para que não ocorra perda da estabilidade hemodinâmica, respiratória e neurológica. Também, pode haver complicações graves no transporte, desde a perda de cateteres venosos, extubação acidental e hipoxemia até a parada cardiorrespiratória, acarretando sérios riscos à saúde do paciente e a necessidade, por vezes, de realizar procedimentos avançados de suporte vital em condições distantes das ideais ${ }^{(12-17)}$. Além disso, a maioria dos trabalhos encontrados na literatura científica se refere a estudos realizados em adultos, sendo escassa a investigação no período neonatal. Vale frisar, neste contexto, a falta de rotinas para o transporte intra-hospitalar em grande parte das unidades neonatais brasileiras, nas quais a possibilidade de intercorrências clínicas durante estes deslocamentos é, em geral, negligenciada.

Os estudos que existem sobre transporte de recém-nascidos versam quase que exclusivamente a respeito do transporte inter-hospitalar ${ }^{(18-20)}$. No Brasil, os relatos existentes se referem a intercorrências clínicas apresentadas por recémnascidos durante o transporte entre diferentes instituições, como o realizado em Florianópolis com 122 transportes neonatais para a UTI do Hospital Infantil Joana de Gusmão em 1992/1993 ${ }^{(18)}$, aquele efetuado em Porto Alegre com 55 recém-nascidos transferidos para a UTI do Hospital Materno-Infantil Presidente Vargas de fevereiro a novembro de $2000^{(19)}$ e o de Recife, com 234 neonatos transferidos para o Berçário do Instituto Materno Infantil de Pernambuco em $2001^{(20)}$. Não foi possível encontrar na literatura outros estudos com amostragem de transporte intra-hospitalar neonatal tão numerosa quanto a aqui apresentada, dificultando a comparação dos resultados obtidos.

O peso ao nascer da população transportada variou de 600 a 5.200 g e a média, nos diferentes anos, foi acima de $1.500 \mathrm{~g}$, pois a maior clientela dos transportes foi a de re- 
cém-nascidos portadores de malformações congênitas, que necessitavam de procedimentos diagnósticos e terapêuticos fora da unidade neonatal e, não, os prematuros extremos. Tal afirmativa é confirmada pelo fato de a idade gestacional média dos pacientes transportados, em todos os anos, se situar ao redor de 35 semanas, idade gestacional na qual há, em diversas ocasiões, interrupção eletiva das gestações de fetos com malformações de sistema nervoso central, renais e com defeitos de fechamento da parede abdominal.

As doenças neurológicas constituíram a principal doença de base que levou à indicação de transporte intra-hospitalar em todos os anos do estudo, destacando-se, dentre elas, as malformações do sistema nervoso central, com especial prevalência de hidrocefalia e defeitos do fechamento do tubo neural. Ao estudar recém-nascidos em uma maternidade terciária, com um centro de medicina fetal bastante atuante, esse achado era esperado. As malformações do sistema nervoso central se constituem na principal indicação para acompanhamento na medicina fetal, sendo a hidrocefalia a de maior prevalência ${ }^{(21)}$.

A freqüência de hipotermia durante e até duas horas após o transporte foi bastante alta, ocorrendo em 87 (17\%) dos 502 transportes estudados, decrescendo no decorrer dos anos de estudo. A comparação deste resultado com a literatura é difícil, pois os trabalhos de transporte intra-hospitalar são realizados com adultos, nos quais a valorização da presença de hipotermia é pequena. Por outro lado, em transportes inter-hospitalares neonatais, a freqüência de hipotermia varia de 15 a $40 \%{ }^{(18-20)}$. O achado pode ser explicado, por um lado, pelas características neonatais próprias que favorecem o surgimento de hipotermia, como: habilidade limitada em produzir calor, extensa superfície corporal em relação ao peso e pouca quantidade de tecido subcutâneo, entre outras ${ }^{(22)}$. Por outro lado, fatores relacionados ao próprio transporte, como sua duração, transporte para ambientes frios (exames de diagnóstico por imagem) podem ter influenciado na freqüência desta intercorrência. De qualquer forma, sabe-se há quase 30 anos que o treinamento e a habilidade da equipe de transporte são fundamentais para evitar a hipotermia ${ }^{(21)}$ e que o aumento da morbidade e da mortalidade relacionadas ao transporte é diretamente proporcional a cada grau de perda de temperatura durante o transporte ${ }^{(23)}$.

Neste contexto, o estudo dos fatores associados à presença de hipotermia na casuística de pacientes transportados aqui apresentada (Tabela 5) confirma a interferência das variáveis acima mencionadas. Assim, foram fatores de risco para a hipotermia: presença de um serviço menos experiente com o transporte intra-hospitalar (primeiro ano do estudo, ou seja, 1997), duração prolongado do procedimento (saída da unidade por período superior a 180 minutos) e presença de malformações congênitas (neonatos com síndromes genéticas e com alterações neurológicas, em sua maioria, portadores de hidrocefalia e/ou defeitos do fechamento de tubo neural). O peso maior do que $1.500 \mathrm{~g}$ mostrou ser um fator protetor para a presença de hipotermia, assim como o fato de ter sido transportado em período mais recente (ano 2000), quando, provavelmente, a experiência dos anos anteriores ajudou a estruturar melhor o serviço em termos de treinamento da equipe. De fato, observou-se uma diminuição da ocorrência de hipotermia durante o transporte no decorrer dos anos de estudo, reforçando que, embora o transporte intra-hospitalar seja um procedimento rotineiro, a presença de equipe treinada e disponibilidade de equipamentos adequados para sua realização se associam a menor morbidade para o paciente.

Houve necessidade de iniciar a oferta de oxigênio inalatório durante o transporte em $3 \%$ dos casos e de iniciar a ventilação mecânica em $8 \%$ dos 502 transportes realizados. Dos últimos, $80 \%$ dos pacientes haviam sido transportados para centro cirúrgicos e intubados para a anestesia e para a realização de procedimentos diversos. O aumento da necessidade de oxigênio e/ou suporte ventilatório para os pacientes durante e até duas horas após transporte foi notado em 33 casos, permanecendo a incidência de tal ocorrência entre 5 e $8 \%$ dos transportes realizados, nos diversos anos do estudo. Dentre os fatores associados à maior necessidade de suporte respiratório (Tabela 6), destaca-se a presença de doenças gastrintestinais e geniturinárias. Este achado se explica pelo fato de se tratarem de pacientes que, em sua maioria, foram transportados com a finalidade cirúrgica e, portanto, saiam da unidade neonatal sem suporte respiratório ou com suporte mínimo e retornavam para fazer a sua recuperação pós-anestésica em ventilação mecânica. Vale ressaltar que, no serviço no qual foi feita esta investigação, a recuperação pós-anestésica de recém-nascidos é realizada na UTI neonatal. Neste sentido, é importante frisar que os pacientes intubados para procedimentos cirúrgicos foram propositalmente incluídos e considerados como portadores de "necessidade de aumento do suporte respiratório", pois, sem dúvida, tais recém-nascidos precisam de uma infra-estrutura diferente ao voltar à unidade, necessidade esta para a qual a equipe que faz o transporte precisa estar preparada. Além disso, pacientes com menor idade gestacional e maior idade pós-natal mostraram risco elevado de precisarem de aumento do suporte respiratório durante e até duas horas após o transporte intrahospitalar, o que sugere se tratarem de pacientes com algum 
grau de displasia broncopulmonar. Nestes recém-nascidos, é provável que o estresse da movimentação e a manipulação inerente ao transporte aumentem a demanda metabólica e a necessidade de oxigênio ${ }^{(16,24)}$.

A principal limitação do presente estudo é o fato de ter sido feito em um único centro e, portanto, refletir uma experiência local, ao longo de um período relativamente amplo (quatro anos), durante o qual as condições de atendimento ao recém-nascido foram gradativamente se modificando. Entretanto, vale ressaltar que se trata do primeiro estudo de transporte intra-hospitalar neonatal brasileiro, com dados obtidos de forma prospectiva e com uma casuística grande.

Os resultados da presente investigação ressaltam a importância de uma equipe treinada para o sucesso do transporte e, neste sentido, a educação continuada dos profissionais de saúde que atuam em UTI é primordial para manter a qualidade do serviço de transporte. A elaboração de novos estudos sobre o tema deve ajudar a estabelecer estratégias para transportar recém-nascidos criticamente doentes com um nível de segurança clínica elevado.

\section{Referências bibliográficas}

1. Venkataraman ST, Orr RA. Intrahospital transport of critically ill patients. Crit Care Clin 1992;8:525-31.

2. Guinsburg R, Vieira ALP. Transporte do recém-nascido com problemas respiratórios. In: Kopelman BI. Clínica de perinatologia: aparelho respiratório em neonatologia, parte 1. $1^{\text {a }}$ ed. São Paulo: Medsi; 2001. p. 169-85.

3. Guinsburg R, Corrêa PPA. Transporte de recém-nascido em insuficiência respiratória. In: Guinsburg R, Kopelman BI, Miyoshi M., editores. Distúrbios respiratórios no período neonatal. $1^{\mathrm{a}}$ ed. São Paulo: Atheneu; 1998. p. 337-50.

4. Guinsburg R, Miyoshi MH, Kolpelman BI. Transporte neonatal. J Pediatr (Rio J) 1992;68:153-8.

5. Das UG, Leuthner SR. Preparing the neonate for transport. Pediatr Clin North Am 2004;51:581-98.

6. Insel J, Weissman C, Kemper M, Askanazi J, Hyman A. Cardiovascular changes during transport of critically ill and postoperative patients. Crit Care Med 1986;14:539-42.

7. Shirley PJ, Bion JF. Intra-hospital transport of critically ill patients: minimising risk. Intensive Care Med 2004;30:1508-10.

8. Venkataraman ST. Intrahospital transport of critically ill children: should we pay attention? Crit Care Med 1999;27:694-5.

9. Gervais HW, Eberle B, Konietzke D, Hennes HJ, Dick W. Comparison of blood gases of ventilated patients during transport. Crit Care Med 1987;15:761-3.

10. Shenai JP, Johnson GE, Varney RV. Mechanical vibration in neonatal transport. Pediatrics 1981;68:55-7.

11. Seshia MK. Temperature control. In: Perlman M, Kirpalani MH, Moore MA, editors. Residents handbook of neonatology. Ontario: BCDecker; 1999. p. 40-8.

12. MacDonald GM. Organization of a neonatal-pediatric interfacility transport service. In: MacDonald MG. Guidelines for air and ground transport of neonatal and pediatric patients. $4^{\text {th }}$ ed. Chicago: American Academy of Pediatrics; 1999. p. 1-65.

13. Edge WE, Kanter RK, Weigle CG. Reduction of morbidity in interhospital transport by specialized pediatric staff. Crit Care Med 1994;22:1186-91.
14. Towers CV, Bonebrake R, Padilla G, Rumney P. The effect of transport on the rate of severe intraventricular hemorrhage in very low birth weight infants. Obstet Gynecol 2000;95:291-5.

15. Segal S. Transfer of a premature or other high risk newborn infants to referral hospital. Pediatr Clin North Am 1996;13:1195-205.

16. Kuch BA, Munoz R, Orr RA, Watson RS. Unplanned transport events and severity of illness: are we conveying the whole picture? Pediatrics 2007;119:648-9.

17. Yeager SB, Horbar JD, Greco KM, Duff J, Thiagarajan RR, Laussen PC Pretransport and posttransport characteristics and outcomes of neonates who were admitted to a cardiac intensive care unit. Pediatrics 2006;118:1070-7.

18. Silva CV, Pereira LD. Transporte neonatal em Santa Catarina. Arq Catarin Med 1995;24:6-11.

19. Vacaro JE, Chazan DT. Condições de transporte dos recém-nascidos na UTI Neonatal do Hospital Materno Infantil Presidente Vargas. Resumo do XVII Congresso Brasileiro de Perinatologia; 10 a 14 de novembro de 2001; Florianópolis (SC). Sociedade Brasileira de Pediatria, 2001:242.

20. Silveira SMM, Mello MJG, Arruda S, Frias PG. Condições de transporte dos recém-nascidos transferidos para unidade de referência em Pernambuco. Resumo do XVII Congresso Brasileiro de Perinatologia; 10 a 14 de novembro de 2001; Florianópolis (SC). Sociedade Brasileira de Pediatria, 2001:242.

21. Zugaib M, Pedreira DA. Malformações do sistema nervoso central. In: Zugaib M, Pedreira DA, Brizot ML. Medicina Fetal. $1^{\text {a }}$ ed. Rio de Janeiro: Atheneu; 1998. p. $175-95$

22. Arad I, Gofin R, Baras M, Bar-Oz B, Peleg O, Epstein L. Neonatal outcome of inborn and transported very low birth weight infants: relevance of perinatal factors. Eur J Obstet Gynecol Reprod Biol 1999;83:151-7.

23. Nichols VG. Neonatal transport. In: Cloherty JP, Stark AR, editors. Manual of neonatal care. $3^{\text {rd }}$ ed. Philadelphia: Lippincott-Raven; 1998. p. 143-9.

24. Clarke TA. A review of neonatal transports. Ir Med J 1985;78:40-3. 